\title{
A new symmetric endomorphism operator for some generalizations of certain generating functions
}

\author{
Ali Boussayoud ${ }^{1}$, Abdelhamid Abderrezzak ${ }^{2}$ \\ and Serkan Araci ${ }^{3}$ \\ ${ }^{1}$ LMAM-Department of Mathematics \\ Mohamed Seddik Ben Yahia University, Jijel, Algeria \\ e-mail: aboussayoudeyahoo.fr \\ ${ }^{2}$ University of Paris 7, LITP \\ Place Jussieu, Paris cedex 05, France \\ e-mail: abdelhamidabderrezzak@gmail.com \\ ${ }^{3}$ Department of Economics, Faculty of Economics, Administrative and Social Sciences \\ Hasan Kalyoncu University, TR-27410, Gaziantep, Turkey \\ e-mail: mtsrkn@hotmail.com
}

Received: 28 February 2018

Accepted: 24 October 2018

\begin{abstract}
In this article, we introduce new symmetric endomorphism operators by making use of appropriate infinite product series. The main results show that after direct calculations, the proposed operators are qualified to obtain generating functions for $k$-Jacobsthal numbers and Tchebychev polynomials of the first and second kind.
\end{abstract}

Keywords: Symmetric functions, Mersenne numbers, $k$-Jacobsthal numbers.

2010 Mathematics Subject Classification: 05A15, 05E05, $11 \mathrm{~B} 39$.

\section{Introduction and preliminaries}

It is well-known in the theory of orthogonal polynomials that a positive definite inner product generates a unique set of real orthogonal polynomials. When the inner product is not Hermitian, the existence of the corresponding orthogonal polynomials is not guaranteed. A generalization of orthogonal polynomials in the sense that they satisfy $r \in \mathbb{N}$ orthogonality conditions leads to the 
concept of multiple orthogonal polynomials. These orthogonal polynomials arise naturally in the theory of simultaneous rational approximation, in particular in Hermite-Padé approximation of a system of $r$ (Markov) functions, see [24].

Recently, Baeder et al. [3] used relations and series rearrangement to generalize generating functions for several higher continuous orthogonal polynomials in the Askey scheme, namely the Wilson, continuous dual Hahn, continuous Hahn, and Meixner-Pollaczek polynomials. For different works in the field, readers may refer to $[9,11,12,14,16,17,19,21-23]$.

In this contribution, we define a new useful operator denoted by $\delta_{e_{1} e_{2}}^{-k}$, for which we can formulate, extend and prove new results based on our previous ones [5-7]. In order to determine generating functions for $k$-Jacobsthal numbers and Mersenne numbers and Tchebychev polynomials of the first and second kind, we combine between our indicated past techniques and these presented polishing approaches.

Definition 1.1 ([1]). Given two sets of indeterminate $A$ and $E$ (called alphabets), we define $S_{j}(A-E)$ as follows:

$$
\frac{\Pi_{e \epsilon B}(1-z e)}{\Pi_{a \epsilon A}(1-z a)}=\sum_{j=0}^{\infty} S_{j}(A-E) z^{j},
$$

with $S_{j}(A-E)=0$ for $j<0$.

Remark 1.1. By taking $A=0$ in (1), we obtain

$$
\Pi_{e \epsilon B}(1-z e)=\sum_{j=0}^{\infty} S_{j}(-E) z^{j} .
$$

Proposition 1.1 ([2]). Considering successively the case of $A=0$ or $E=0$, we can derive the following factorization

$$
\sum_{j=0}^{\infty} S_{j}(A-E) z^{j}=\sum_{j=0}^{\infty} S_{j}(A) z^{j} \sum_{j=0}^{\infty} S_{j}(-E) z^{j}
$$

Thus,

$$
S_{n}(A-E)=\sum_{k=0}^{n} S_{n-k}(A) S_{k}(-E) .
$$

The summation is in fact limited to a finite number of nonzero terms. In particular, we have $\Pi_{e \in B}(x-e)=S_{n}(x-E)=S_{0}(-E) x^{n}+S_{1}(-E) x^{n-1}+S_{2}(-E) x^{n-2}+\cdots+S_{n}(-E)$, where $S_{j}(-E)$ are the coefficients of polynomials $S_{n}(x-E)$ for $0<j<n$. We note that $S_{j}(-E)=0$ for $j>n$.

For $E=\{e, e, \ldots\}$ (we denote $E=n e$ ), we have

$$
S_{n}(x-n e)=(x-e)^{n} .
$$

Thus, the special case of $E=\{1, \ldots, 1\}$ gives the two binomial coefficients

$$
S_{j}(-n)=(-1)^{j}\left(\begin{array}{l}
n \\
j
\end{array}\right) \text { and } S_{j}(n)=\left(\begin{array}{c}
n+j-1 \\
j
\end{array}\right) \text {. }
$$


By combining (4) and (5), we obtain the following expression

$$
S_{j}(A-j x)=S_{j}(A)-\left(\begin{array}{l}
j \\
1
\end{array}\right) S_{j-1}(A) x+\cdots+(-1)^{j}\left(\begin{array}{l}
j \\
j
\end{array}\right) x^{j} .
$$

Definition 1.2 ([20]). Given a function $g$ on $\mathbb{R}^{n}$, the divided difference operator is defined as follows:

$$
\partial_{x_{i} x_{i+1}}(g)=\frac{g\left(x_{1}, \ldots, x_{i}, x_{i+1}, \ldots, x_{n}\right)-g\left(x_{1}, \ldots, x_{i-1}, x_{i+1}, x_{i}, x_{i+2}, \ldots, x_{n}\right)}{x_{i}-x_{i+1}} .
$$

Definition 1.3 ([4]). The symmetrizing operator $\delta_{e_{1} e_{2}}^{k}$ is defined by

$$
\delta_{e_{1} e_{2}}^{k}\left(e_{1}^{j}\right)=\frac{e_{1}^{k+j}-e_{2}^{k+j}}{e_{1}-e_{2}}=S_{k+j-1}\left(e_{1}+e_{2}\right), \text { for all } k, j \in \mathbb{N} .
$$

Definition 1.4 ([5]). The symmetrizing operator $\delta_{e_{1} e_{2}}^{-k}$ is defined by

$$
\delta_{e_{1} e_{2}}^{-k} f=\frac{e_{2}^{k} f(x)-e_{1}^{k} f(y)}{\left(e_{1} e_{2}\right)^{k}\left(e_{1}-e_{2}\right)}, \text { for all } k \in \mathbb{N} .
$$

\section{Main results}

Lemma 2.1 ([5]). Let $E=\left\{e_{1}, e_{2}\right\}$, we define the operator $\delta_{e_{1} e_{2}}^{-k}$ as follows:

$$
\delta_{e_{1} e_{2}}^{-k} f\left(e_{1}\right)=\frac{-S_{k-1}(E)}{e_{1}^{k} e_{2}^{k}} f\left(e_{1}\right)+\frac{e_{1}^{k}}{e_{1}^{k} e_{2}^{k}} \partial_{e_{1} e_{2}} f\left(e_{1}\right), \text { for all } k \in \mathbb{N} .
$$

Theorem 2.1. Given an alphabet $E=\left\{e_{1}, e_{2}\right\}$, two sequences $\sum_{j=0}^{+\infty} a_{j} z^{j}$ and $\sum_{j=0}^{+\infty} b_{j} z^{j}$ such that $\left(\sum_{j=0}^{+\infty} a_{j} z^{j}\right)\left(\sum_{j=0}^{+\infty} b_{j} z^{j}\right)=1$, then

$$
\frac{\sum_{j=0}^{\infty} b_{j} \delta_{e_{1} e_{2}}^{k}\left(e_{1}^{j}\right) z^{j}}{\left(\sum_{j=0}^{\infty} b_{j} e_{1}^{j} z^{j}\right)\left(\sum_{j=0}^{\infty} b_{j} e_{2}^{j} z^{j}\right)}=\sum_{j=0}^{k-1} a_{j} e_{1}^{j} e_{2}^{j} \delta_{e_{1} e_{2}}^{k-j}\left(e_{1}\right) z^{j}-e_{1}^{k} e_{2}^{k} z^{k+1} \sum_{j=0}^{\infty} a_{j+k+1} \delta_{e_{1} e_{2}}^{k+1}\left(e_{1}\right) z^{j}
$$

Proof. Let $\sum_{j=0}^{\infty} a_{j} z^{j}$ and $\sum_{j=0}^{\infty} b_{j} z^{j}$ be two sequences such that $\sum_{j=0}^{\infty} a_{j} z^{j} \sum_{j=0}^{\infty} b_{j} z^{j}=1$. The left-hand side of (6) can be written as

$$
\frac{-S_{k-1}(E)}{e_{1}^{k} e_{2}^{k}} f\left(e_{1}\right)+\frac{e_{1}^{k}}{e_{1}^{k} e_{2}^{k}} \partial_{e_{1} e_{2}} f\left(e_{1}\right)=\frac{-S_{k-1}(E)}{e_{1}^{k} e_{2}^{k}} \frac{1}{\sum_{j=0}^{\infty} b_{j} e_{1}^{j} z^{j}}+\frac{e_{1}^{k}}{e_{1}^{k} e_{2}^{k}} \partial_{e_{1} e_{2}} \frac{1}{\sum_{j=0}^{\infty} b_{j} e_{1}^{j} z^{j}}
$$




$$
\begin{aligned}
& =\frac{1}{e_{1}^{k} e_{2}^{k}}\left(\frac{-\sum_{j=0}^{\infty} b_{j} e_{2}^{j} S_{k-1}(E) z^{j}+\frac{e_{1}^{k}}{e_{1}-e_{2}} \sum_{j=0}^{\infty} b_{j}\left(e_{2}^{j}-e_{1}^{j}\right) z^{j}}{\left(\sum_{j=0}^{\infty} b_{j} e_{1}^{j} z^{j}\right)\left(\sum_{j=0}^{\infty} b_{j} e_{2}^{j} z^{j}\right)}\right) \\
& =\frac{-1}{e_{1}^{k} e_{2}^{k}}\left(\frac{\sum_{j=0}^{\infty} b_{j}\left(e_{2}^{j} e_{1}^{k}-e_{2}^{k}\right.}{e_{1}-e_{2}}+e_{1}^{k} e_{1}^{e_{1}^{j}-e_{2}^{j}}\right) z^{j} \\
& =\frac{-1}{e_{1}^{k} e_{2}^{k}}\left(\frac{\left.\sum_{j=0}^{\infty} b_{j} e_{1}^{j} z^{j}\right)\left(\sum_{j=0}^{\infty} b_{j} e_{2}^{j} z^{j}\right)}{\left.\left(\sum_{j=0}^{\infty} b_{j} e_{1}^{j} z^{j}\right)\left(\sum_{j=0}^{k+j} b_{2}^{k+e_{2}}\right) z_{2}^{j} z^{j}\right)}\right) \\
& =\frac{-1}{e_{1}^{k} e_{2}^{k}}\left(\frac{\sum_{j=0}^{\infty} b_{j} \delta_{e_{1} e_{2}}^{k}\left(e_{1}^{j}\right) z^{j}}{\left(\sum_{j=0}^{\infty} b_{j} e_{1}^{j} z^{j}\right)\left(\sum_{j=0}^{\infty} b_{j} e_{2}^{j} z^{j}\right)}\right) .
\end{aligned}
$$

The right-hand side can be expressed as

$$
\begin{aligned}
\delta_{e_{1} e_{2}}^{-k} f\left(e_{1}\right) & =\delta_{e_{1} e_{2}}^{-k}\left(\sum_{j=0}^{\infty} a_{j} e_{1}^{j} z^{j}\right) \\
& =\frac{e_{2}^{k} \sum_{j=0}^{\infty} a_{j} e_{1}^{j} z^{j}-e_{1}^{k} \sum_{j=0}^{\infty} a_{j} e_{2}^{j} z^{j}}{e_{1}^{k} e_{2}^{k}\left(e_{1}-e_{2}\right)} \\
& =\frac{1}{e_{1}^{k} e_{2}^{k}}\left(\sum_{j=0}^{\infty} a_{j} \frac{e_{2}^{k} e_{1}^{j}-e_{1}^{k} e_{2}^{j}}{e_{1}-e_{2}} z^{j}\right) \\
& =\frac{1}{e_{1}^{k} e_{2}^{k}}\left(\sum_{j=0}^{k-1} a_{j} \frac{e_{2}^{k} e_{1}^{j}-e_{1}^{k} e_{2}^{j}}{e_{1}-e_{2}} z^{j}+\sum_{j=k+1}^{\infty} a_{j} \frac{e_{2}^{k} e_{1}^{j}-e_{1}^{k} e_{2}^{j}}{e_{1}-e_{2}} z^{j}\right) \\
& =\frac{-1}{e_{1}^{k} e_{2}^{k}}\left(\sum_{j=0}^{k-1} a_{j} e_{1}^{j} e_{2}^{j} \delta_{e_{1} e_{2}}^{k-j}\left(e_{1}\right) z^{j}-e_{1}^{k} e_{2}^{k} z^{k+1} \sum_{j=0}^{\infty} a_{j+k+1} \delta_{e_{1} e_{2}}^{k+1}\left(e_{1}\right) z^{j}\right),
\end{aligned}
$$

therefore

$$
\frac{\sum_{j=0}^{\infty} b_{j} \delta_{e_{1} e_{2}}^{k}\left(e_{1}^{j}\right) z^{j}}{\left(\sum_{j=0}^{\infty} b_{j} e_{1}^{j} z^{j}\right)\left(\sum_{j=0}^{\infty} b_{j} e_{2}^{j} z^{j}\right)}=\sum_{j=0}^{k-1} a_{j} e_{1}^{j} e_{2}^{j} \delta_{e_{1} e_{2}}^{k-j}\left(e_{1}\right) z^{j}-e_{1}^{k} e_{2}^{k} z^{k+1} \sum_{j=0}^{\infty} a_{j+k+1} \delta_{e_{1} e_{2}}^{k+1}\left(e_{1}\right) z^{j} .
$$


Theorem 2.2. Given two alphabets $E=\left\{e_{1}, e_{2}\right\}$ and $A=\left\{a_{1}, a_{2}, \ldots, a_{n}\right\}$, we have

$$
\begin{aligned}
\frac{\sum_{j=0}^{\infty} S_{j}(-A) \delta_{e_{1} e_{2}}^{k}\left(e_{1}^{j}\right) z^{j}}{\left(\sum_{j=0}^{\infty} S_{j}(-A) e_{1}^{j} z^{j}\right)\left(\sum_{j=0}^{\infty} S_{j}(-A) e_{2}^{j} z^{j}\right)} & =\sum_{j=0}^{k-1} S_{j}(A) e_{1}^{j} e_{2}^{j} \delta_{e_{1} e_{2}}^{k-j}\left(e_{1}\right) z^{j} \\
& -e_{1}^{k} e_{2}^{k} z^{k+1} \sum_{j=0}^{\infty} S_{j+k+1}(A) \delta_{e_{1} e_{2}}^{k+1}\left(e_{1}\right) z^{j},
\end{aligned}
$$

for all $k \in \mathbb{N}$.

Proof. The action of the operator $\delta_{e_{1} e_{2}}^{-k}$ on the series $f\left(e_{1}\right)=\left(\sum_{j=0}^{\infty} S_{j}(-A) e_{1}^{j} z^{j}\right)^{-1}$ gives the left member of equality (7), then

$$
\begin{aligned}
\delta_{e_{1} e_{2}}^{-k} f\left(e_{1}\right) & =\delta_{e_{1} e_{2}}^{-k}\left(\frac{1}{\sum_{j=0}^{\infty} S_{j}(-A) e_{1}^{j} z^{j}}\right) \\
& =\frac{\frac{e_{2}^{\infty} S_{j}(-A) e_{1}^{j} z^{j}}{\sum_{j=0}^{\infty} S_{j}(-A) e_{2}^{j} z^{j}}}{e_{1}^{k} e_{2}^{k}\left(e_{1}-e_{2}\right)} \\
& =\frac{\sum_{j=0}^{\infty} S_{j}(-A) e_{2}^{j+k} z^{j}-\sum_{j=0}^{\infty} S_{j}(-A) e_{1}^{j+k} z^{j}}{e_{1}^{k} e_{2}^{k}\left(e_{1}-e_{2}\right)\left(\sum_{j=0}^{\infty} S_{j}(-A) e_{1}^{j} z^{j}\right)\left(\sum_{j=0}^{\infty} S_{j}(-A) e_{2}^{j} z^{j}\right)} \\
= & \frac{-1}{e_{1}^{k} e_{2}^{k}}\left(\frac{\sum_{j=0}^{\infty} S_{j}(-A) \delta_{e_{1} e_{2}}^{k}\left(e_{1}^{j}\right) z^{j}}{\left(\sum_{j=0}^{\infty} S_{j}(-A) e_{1}^{j} z^{j}\right)\left(\sum_{j=0}^{\infty} S_{j}(-A) e_{2}^{j} z^{j}\right)}\right) .
\end{aligned}
$$

The second member of the formula (7) is written as:

$$
\begin{aligned}
\delta_{e_{1} e_{2}}^{-k} f\left(e_{1}\right) & =\delta_{e_{1} e_{2}}^{-k}\left(\sum_{j=0}^{\infty} S_{j}(A) e_{1}^{j} z^{j}\right) \\
& =\frac{e_{2}^{k} \sum_{j=0}^{\infty} S_{j}(A) e_{1}^{j} z^{j}-e_{1}^{k} \sum_{j=0}^{\infty} S_{j}(A) e_{2}^{j} z^{j}}{e_{1}^{k} e_{2}^{k}\left(e_{1}-e_{2}\right)} \\
& =\frac{1}{e_{1}^{k} e_{2}^{k}}\left(\sum_{j=0}^{\infty} S_{j}(A) \frac{e_{2}^{k} e_{1}^{j}-e_{1}^{k} e_{2}^{j}}{e_{1}-e_{2}} z^{j}\right) \\
& =\frac{1}{e_{1}^{k} e_{2}^{k}}\left(\sum_{j=0}^{k-1} S_{j}(A) \frac{e_{2}^{k} e_{1}^{j}-e_{1}^{k} e_{2}^{j}}{e_{1}-e_{2}} z^{j}+\sum_{j=k+1}^{\infty} S_{j}(A) \frac{e_{2}^{k} e_{1}^{j}-e_{1}^{k} e_{2}^{j}}{e_{1}-e_{2}} z^{j}\right)
\end{aligned}
$$




$$
=\frac{-1}{e_{1}^{k} e_{2}^{k}}\left(\sum_{j=0}^{k-1} S_{j}(A) e_{1}^{j} e_{2}^{j} \delta_{e_{1} e_{2}}^{k-j}\left(e_{1}\right) z^{j}-e_{1}^{k} e_{2}^{k} z^{k+1} \sum_{j=0}^{\infty} S_{j+k+1}(A) \delta_{e_{1} e_{2}}^{k+1} z^{j}\right) .
$$

The two quantities are equal.

\section{Applications}

\subsection{Applications of Theorem 2.1}

3.1.1 The case of $\frac{1}{1-z}=\sum_{j=0}^{\infty} z^{j}$

Corollary 3.1. Given an alphabet $E=\left\{e_{1}, e_{2}\right\}$, we have

$$
\frac{S_{k-1}(E)-S_{k}(E)}{\left(1-e_{1} z\right)\left(1-e_{2} z\right)}=\sum_{j=0}^{k-1} e_{1}^{j} e_{2}^{j} S_{k-j-1}(E) z^{j}-e_{1}^{k} e_{2}^{k} z^{k+1} \sum_{j=0}^{\infty} S_{j}(E) z^{j}, \text { for all } k \in \mathbb{N} \text {. }
$$

For $\mathbf{k}=\mathbf{1}$ in (8), we have

$$
\sum_{j=0}^{\infty} S_{j}(E) z^{j}=\frac{1}{\left(1-e_{1} z\right)\left(1-e_{2} z\right)}=\frac{1}{1-\left(e_{1}+e_{2}\right) z+e_{1} e_{2} z^{2}} .
$$

We replace $e_{2}$ by $\left(-e_{2}\right)$ in $(9)$, we obtain

$$
\sum_{j=0}^{\infty} S_{j}\left(e_{1}+\left[-e_{2}\right]\right) z^{j}=\frac{1}{1-\left(e_{1}-e_{2}\right) z-e_{1} e_{2} z^{2}} .
$$

Setting $\left\{\begin{array}{c}e_{1} e_{2}=2 \\ e_{1}-e_{2}=k\end{array}\right.$ in (10), and this gives

$$
\sum_{j=0}^{\infty} S_{j-1}\left(e_{1}+\left[-e_{2}\right]\right) z^{j}=\frac{z}{1-k z-2 z^{2}}=\sum_{j=0}^{\infty} J_{k, j} z^{j},
$$

which represents a generating function for $k$-Jacobsthal numbers, such that

$$
J_{k, j}=S_{j-1}\left(e_{1}+\left[-e_{2}\right]\right),
$$

with $e_{1,2}=\frac{k \pm \sqrt{k^{2}+8}}{2}$.

Again, we put $\left\{\begin{array}{c}e_{1} e_{2}=-2 \\ e_{1}-e_{2}=3\end{array}\right.$ in (10), we get

$$
\sum_{j=0}^{\infty} S_{j-1}\left(e_{1}+\left[-e_{2}\right]\right) z^{j}=\frac{z}{1-3 z+2 z^{2}},
$$

which represents a generating function for Mersenne numbers, such that $M_{j}=S_{j-1}\left(e_{1}+\left[-e_{2}\right]\right)$, with $e_{1}=2, e_{2}=1$. 
Theorem 3.1. We have the following new generating function of the $k$-Jacobsthal numbers at negative indices as

$$
\sum_{j=0}^{\infty} J_{k,-j} z^{j}=\frac{z}{1+k z-2 z^{2}}
$$

Theorem 3.2. We have the following new generating function of the Mersenne numbers at negative indices as

$$
\sum_{j=0}^{\infty} M_{-j} z^{j}=\frac{z}{1+3 z+2 z^{2}}
$$

Furthermore, we replace $e_{1}$ by $2 e_{1}$ and $e_{2}$ by $\left(-2 e_{2}\right)$ in (9), and under the condition $4 e_{1} e_{2}=-1$, we obtain

$$
\sum_{j=0}^{+\infty} S_{j}\left(2 e_{1}+\left[-2 e_{2}\right]\right) z^{j}=\frac{1}{1-2\left(e_{1}-e_{2}\right) z+z^{2}}
$$

which represents a generating function for Tchebychev polynomials of the second kind given by Boussayoud et al. [4,8], such that $U_{j}=S_{j}\left(2 e_{1}+\left[-2 e_{2}\right]\right)$. Moreover, from formula (13), we can deduce that

$$
\sum_{j=0}^{+\infty}\left[S_{j}\left(2 e_{1}+\left[-2 e_{2}\right]\right)-\left(e_{1}-e_{2}\right) S_{j-1}\left(2 e_{1}+\left[-2 e_{2}\right]\right)\right] z^{j}=\frac{1-\left(e_{1}-e_{2}\right) z}{1-2\left(e_{1}-e_{2}\right) z+z^{2}}
$$

which represents a generating function for Tchebychev polynomials of the first kind given in $[4,7]$, such that

$$
T_{j}\left(e_{1}-e_{2}\right)=\left[S_{j}\left(2 e_{1}+\left[-2 e_{2}\right]\right)-\left(e_{1}-e_{2}\right) S_{j-1}\left(2 e_{1}+\left[-2 e_{2}\right]\right)\right] .
$$

For $\mathbf{k}=\mathbf{2}$ in (8), we have

$$
\sum_{j=0}^{\infty} S_{j+1}(E) z^{j}=\frac{e_{1}+e_{2}-e_{1} e_{2} z}{\left(1-z e_{1}\right)\left(1-z e_{2}\right)}=\frac{e_{1}+e_{2}-e_{1} e_{2} z}{1-\left(e_{1}+e_{2}\right) z+e_{1} e_{2} z^{2}} .
$$

Also, by replacing $e_{2}$ with $\left(-e_{2}\right)$ in (14), we obtain

$$
\sum_{j=0}^{\infty} S_{j+1}\left(e_{1}+\left[-e_{2}\right]\right) z^{j}=\frac{\left(e_{1}-e_{2}\right)+e_{1} e_{2} z}{1-\left(e_{1}-e_{2}\right) z-e_{1} e_{2} z^{2}}
$$

Putting $\left\{\begin{array}{c}e_{1} e_{2}=2 \\ e_{1}-e_{2}=k\end{array}\right.$ in (15), it gives

$$
\sum_{j=0}^{\infty} S_{j+1}\left(e_{1}+\left[-e_{2}\right]\right) z^{j}=\frac{k+2 z}{1-k z-2 z^{2}}
$$

which represents a new generating function. 
Substituting $\left\{\begin{array}{c}e_{1} e_{2}=1 \\ e_{1}-e_{2}=k\end{array}\right.$ in (15), yields

$$
\sum_{j=0}^{\infty} S_{j+1}\left(e_{1}+\left[-e_{2}\right]\right) z^{j}=\frac{k+z}{1-k z-z^{2}}
$$

which represents a new generating function.

Multiplying the equation (10) by (2) and subtracting it from (11) by $(k)$, we obtain

$$
\sum_{j=0}^{\infty}\left(2 S_{j}\left(e_{1}+\left[-e_{2}\right]\right)-k S_{j-1}\left(e_{1}+\left[-e_{2}\right]\right)\right) z^{j}=\frac{2-k z}{1-k z-2 z^{2}},
$$

which represents a generating function for $k$-Jacobsthal-Lucas numbers such that

$$
j_{k, j}=2 S_{j}\left(e_{1}+\left[-e_{2}\right]\right)-k S_{j-1}\left(e_{1}+\left[-e_{2}\right]\right) .
$$

Theorem 3.3. We have the following new generating function of the $k$-Jacobsthal numbers at negative indices as

$$
\sum_{j=0}^{\infty} j_{k,-j} z^{j}=\frac{2+k z}{1+k z-2 z^{2}}
$$

\subsection{Applications of Theorem 2.2}

In this section, we attempt to give results for some well-known generating functions. In fact, we will use Theorem 2.2 to derive $k$-Jacobsthal numbers and Tchebychev polynomials of second kind. If $k=1$, the next Corollary gives a new generating function.

Corollary 3.2. Given two alphabets $E=\left\{e_{1}, e_{2}\right\}$ and $A=\left\{a_{1}, a_{2},\right\}$, we have

$$
\begin{aligned}
& \sum_{j=0}^{\infty} S_{j+2}\left(a_{1}+a_{2}\right) S_{j}\left(e_{1}+e_{2}\right) z^{j} \\
& =\frac{e_{1} e_{2} a_{1}^{2} a_{2}^{2} z^{2}-a_{1} a_{2}\left(e_{1}+e_{2}\right)\left(a_{1}+a_{2}\right) z+\left(a_{1}+a_{2}\right)^{2}-a_{1} a_{2}}{\prod_{a \in A_{2}}\left(1-a e_{1} z\right) \prod_{a \in A_{2}}\left(1-a e_{2} z\right)} .
\end{aligned}
$$

\subsubsection{The case $E=\{1, y\}, A=\{1, x\}$}

Substituting $e_{1}=a_{1}=1, e_{2}=x$ and $a_{2}=y$ in (18), we obtain

$$
\sum_{j=0}^{\infty} S_{j+2}(1+x) S_{j}(1+y) z^{j}=\frac{x y^{2} z^{2}-x(1+x)(1+y) z+(1+x)^{2}-x}{(1-z)(1-x z)(1-y z)(1-x y z)}
$$

which represents a new generating function.

Proposition 3.1. For all $j \in \mathbb{N}$, we have

$$
S_{j+2}(1+x)=S_{j}(1+x)+x(x+1) S_{j}(x) .
$$


Proof. From (19), we have

$$
\begin{aligned}
\sum_{j=0}^{\infty} S_{j+2}(1+x) S_{j}(1+y) z^{j} & =\frac{x y^{2} z^{2}-x(1+x)(1+y) z+(1+x)^{2}-x}{(1-z)(1-z x)(1-z y)(1-z x y)} \\
& =\frac{1-x y z^{2}}{(1-z)(1-z x)(1-z y)(1-z x y)}+\frac{x(1+x)}{(1-z x)(1-z x y)}
\end{aligned}
$$

after that, as in $[7,18]$, we have $\sum_{j=0}^{\infty} S_{j}(1+x) S_{j}(1+y) z^{j}=\frac{1-x y z^{2}}{(1-z)(1-z x)(1-z y)(1-z x y)}$, then

$$
\sum_{j=0}^{\infty} S_{j+2}(1+x) S_{j}(1+y) z^{j}=\sum_{j=0}^{\infty} S_{j}(1+x) S_{j}(1+y) z^{j}+\sum_{j=0}^{\infty} x(1+x) S_{j}(x) S_{j}(1+y) z^{j}
$$

therefore

$$
S_{j+2}(1+x)=S_{j}(1+x)+x(x+1) S_{j}(x) .
$$

3.2.2 The case $E=\left\{e_{1},-e_{2}\right\}, A=\left\{a_{1},-a_{2}\right\}$

Replacing $e_{2}$ by $\left(-e_{2}\right)$ and $a_{2}$ by $\left(-a_{2}\right)$ in (18) yields

$$
\begin{aligned}
& \sum_{j=0}^{\infty} S_{j+1}\left(a_{1}+\left[-a_{2}\right]\right) S_{j-1}\left(e_{1}+\left[-e_{2}\right]\right) z^{j} \\
& =\frac{-e_{1} e_{2} a_{1}^{2} a_{2}^{2} z^{3}+a_{1} a_{2}\left(e_{1}-e_{2}\right)\left(a_{1}-a_{2}\right) z^{2}+\left(\left(a_{1}-a_{2}\right)^{2}+a_{1} a_{2}\right) z}{\left(1-a_{1} e_{1} z\right)\left(1+a_{2} e_{1} z\right)\left(1+a_{1} e_{2} z\right)\left(1-a_{2} e_{2} z\right)} .
\end{aligned}
$$

This case consists of three related parts.

Firstly, the substitutions of

$$
\left\{\begin{array} { l } 
{ a _ { 1 } - a _ { 2 } = k , } \\
{ a _ { 1 } a _ { 2 } = 2 , }
\end{array} \text { and } \left\{\begin{array}{l}
e_{1}-e_{2}=k \\
e_{1} e_{2}=2
\end{array}\right.\right.
$$

in (20) give

$$
\begin{aligned}
& \sum_{j=0}^{\infty} S_{j+1}\left(a_{1}+\left[-a_{2}\right]\right) S_{j-1}\left(e_{1}+\left[-e_{2}\right]\right) z^{j} \\
& =\frac{\left(k^{2}+2\right) z+2 k^{2} z^{2}-8 z^{3}}{1-k^{2} z-4\left(k^{2}+2\right) z^{2}--4 k^{2} z^{3}+16 z^{4}} \\
& =\sum_{j=0}^{\infty} J_{k, j+2} J_{k, j} z^{j}
\end{aligned}
$$

which represents a new generating function of $k$-Jacobsthal numbers $J_{k, j}$, such that

$$
J_{k, j+2} J_{k, j}=S_{j+1}\left(a_{1}+\left[-a_{2}\right]\right) S_{j-1}\left(e_{1}+\left[-e_{2}\right]\right) .
$$

We have the following theorem.

Theorem 3.4. For $n, k \in \mathbb{N}$, the new generating function of the product of $k$-Jacobsthal numbers is given by 


$$
\sum_{j=0}^{\infty} J_{k, j+1} J_{k, j} z^{j}=\frac{k z+2 k z^{2}}{1-k^{2} z-4\left(k^{2}+2\right) z^{2}--4 k^{2} z^{3}+16 z^{4}} .
$$

Proof. We have

$$
\begin{aligned}
\sum_{j=0}^{\infty} J_{k, j+2} J_{k, j} z^{j} & =\sum_{j=0}^{\infty}\left(k J_{k, j+1}+2 J_{k, j}\right) J_{k, j} z^{j} \\
& =k \sum_{j=0}^{\infty} J_{k, j+1} J_{k, j} z^{j}+2 \sum_{j=0}^{\infty} J_{k, j}^{2} z^{j}
\end{aligned}
$$

since

$$
\sum_{j=0}^{\infty} J_{k, j}^{2} z^{j}=\frac{z-4 z^{3}}{1-k^{2} z-4\left(k^{2}+2\right) z^{2}--4 k^{2} z^{3}+16 z^{4}}, \text { (see [10]) }
$$

we have

$$
\begin{aligned}
\sum_{j=0}^{\infty} J_{k, j+2} J_{k, j} z^{j} & =k \frac{k z+2 k z^{2}}{1-k^{2} z-4\left(k^{2}+2\right) z^{2}--4 k^{2} z^{3}+16 z^{4}} \\
& +\frac{2 z-8 z^{3}}{1-k^{2} z-4\left(k^{2}+2\right) z^{2}--4 k^{2} z^{3}+16 z^{4}},
\end{aligned}
$$

therefore

$$
\sum_{j=0}^{\infty} J_{k, j+1} J_{k, j} z^{j}=\frac{k z+2 k z^{2}}{1-k^{2} z-4\left(k^{2}+2\right) z^{2}--4 k^{2} z^{3}+16 z^{4}} .
$$

Thus, this completes the proof.

Secondly, by making the following restrictions: $e_{1}-e_{2}=1, e_{1} e_{2}=1,4 a_{1} a_{2}=-1$, and by replacing $\left(a_{1}-a_{2}\right)$ by $2\left(a_{1}-a_{2}\right)$ in $(20)$, we get a new generating function, involving the product of $k$-Jacobsthal numbers with Tchebychev polynomial of second kind as follows:

$$
\begin{aligned}
\sum_{j=0}^{\infty} S_{j+1}\left(2 a_{1}+\left[-2 a_{2}\right]\right) S_{j-1}\left(e_{1}+\left[-e_{2}\right]\right) z^{j} & =\frac{\left(4\left(a_{1}-a_{2}\right)^{2}-1\right) z-2 k\left(a_{1}-a_{2}\right) z^{2}-2 z^{3}}{P_{U J}} \\
& =\sum_{j=0}^{\infty} U_{j+1}\left(a_{1}-a_{2}\right) J_{k, j} z^{j},
\end{aligned}
$$

with $P_{U J}=1-2 k\left(a_{1}-a_{2}\right) z+\left(8\left(a_{1}-a_{2}\right)^{2}-k^{2}-4\right) z^{2}+4 k\left(a_{1}-a_{2}\right) z^{3}+4 z^{4}$.

Thirdly, choose $a_{i}$ and $e_{i}(i=1,2)$ such that $4 e_{1} e_{2}=-1,4 a_{1} a_{2}=-1$, and by replacing $\left(a_{1}-a_{2}\right)$ with $2\left(a_{1}-a_{2}\right)$, and $\left(e_{1}-e_{2}\right)$ with $2\left(e_{1}-e_{2}\right)$ in $(20)$, we get a new generating function, involving the square of Tchebychev polynomials of second kind given by

$$
\begin{aligned}
\sum_{j=0}^{\infty} S_{j+2}\left(2 a_{1}+\left[-2 a_{2}\right]\right) S_{j}\left(2 e_{1}+\left[-2 e_{2}\right]\right) z^{j} & =\frac{z^{2}-1+4\left(a_{1}-a_{2}\right)^{2}-4\left(a_{1}-a_{2}\right)\left(e_{1}-e_{2}\right) z}{P_{U U}} \\
& =\sum_{j=0}^{\infty} U_{j+2}\left(a_{1}-a_{2}\right) U_{j}\left(e_{1}-e_{2}\right) z^{j},
\end{aligned}
$$

with $P_{U U}=1-4\left(e_{1}-e_{2}\right)\left(a_{1}-a_{2}\right) z+\left(4\left(a_{1}-a_{2}\right)^{2}+4\left(e_{1}-e_{2}\right)^{2}-2\right) z^{2}-4\left(e_{1}-e_{2}\right)\left(a_{1}-a_{2}\right) z^{3}+z^{4}$. Thus, we get the following theorem. 
Theorem 3.5. We have a new generating function of the product of Tchebychev polynomials of second kind as

$$
\sum_{j=0}^{\infty} U_{j+1}\left(a_{1}-a_{2}\right) U_{j}\left(e_{1}-e_{2}\right) z^{j}=\frac{2\left(a_{1}-a_{2}\right)-2\left(e_{1}-e_{2}\right) z}{P_{U U}} .
$$

Proof. We have,

$$
\begin{array}{r}
\sum_{j=0}^{\infty} U_{j+1}\left(a_{1}-a_{2}\right) U_{j}\left(e_{1}-e_{2}\right) z^{j}=\sum_{j=0}^{\infty}\left[2\left(a_{1}-a_{2}\right) U_{j+1}\left(a_{1}-a_{2}\right)-U_{j}\left(a_{1}-a_{2}\right)\right] U_{j}\left(e_{1}-e_{2}\right) z^{j} \\
=2\left(a_{1}-a_{2}\right) \sum_{j=0}^{\infty} U_{j+1}\left(a_{1}-a_{2}\right) U_{j}\left(e_{1}-e_{2}\right) z^{j}-\sum_{j=0}^{\infty} U_{j}\left(a_{1}-a_{2}\right) U_{j}\left(e_{1}-e_{2}\right) z^{j} .
\end{array}
$$

Since

$$
\sum_{j=0}^{\infty} U_{j}\left(a_{1}-a_{2}\right) U_{j}\left(e_{1}-e_{2}\right) z^{j}=\frac{1-z^{2}}{P_{U U}} \quad(\text { see }[6,7])
$$

so

$$
\sum_{j=0}^{\infty} U_{j+2}\left(a_{1}-a_{2}\right) U_{j}\left(e_{1}-e_{2}\right) z^{j}=2\left(a_{1}-a_{2}\right) \frac{2\left(a_{1}-a_{2}\right)-2\left(e_{1}-e_{2}\right) z}{P_{U U}}-\frac{1-z^{2}}{P_{U U}}
$$

therefore,

$$
\sum_{j=0}^{\infty} U_{j+1}\left(a_{1}-a_{2}\right) U_{j}\left(e_{1}-e_{2}\right) z^{j}=\frac{2\left(a_{1}-a_{2}\right)-2\left(e_{1}-e_{2}\right) z}{P_{U U}} .
$$

This completes the proof.

Finally, according the formulas (9) and (13) in [4,7], and to the fact that

$$
S_{j+1}\left(2 a_{1}+\left[-2 a_{2}\right]\right)=\frac{\left(2 a_{1}\right)^{j+2}-\left(-2 a_{2}\right)^{j+2}}{2 a_{1}+2 a_{2}}
$$

we have

$$
\sum_{j=0}^{\infty} T_{j+2}\left(a_{1}-a_{2}\right) U_{j}\left(e_{1}-e_{2}\right) z^{j}=\frac{-1+2\left(a_{1}-a_{2}\right)^{2}-2\left(a_{1}-a_{2}\right)\left(e_{1}-e_{2}\right) z+z^{2}}{P_{T U}},
$$

with $P_{T U}=1-4\left(e_{1}-e_{2}\right)\left(a_{1}-a_{2}\right) z+\left(4\left(a_{1}-a_{2}\right)^{2}+4\left(e_{1}-e_{2}\right)^{2}-2\right) z^{2}-4\left(e_{1}-e_{2}\right)\left(a_{1}-a_{2}\right) z^{3}+z^{4}$, which represents a new generating function for the combined Tchebychev polynomials of the second and first kinds.

On the other hand, we have

$$
\begin{aligned}
\sum_{j=0}^{\infty} T_{j+2}\left(a_{1}-a_{2}\right) U_{j}\left(e_{1}-e_{2}\right) z^{j} & =\sum_{j=0}^{\infty}\left[2\left(a_{1}-a_{2}\right) T_{j+1}\left(a_{1}-a_{2}\right)-T_{j}\left(a_{1}-a_{2}\right)\right] U_{j}\left(e_{1}-e_{2}\right) z^{j} \\
& =2\left(a_{1}-a_{2}\right) \sum_{j=0}^{\infty} T_{j+1}\left(a_{1}-a_{2}\right) U_{j}\left(e_{1}-e_{2}\right) z^{j} \\
& -\sum_{j=0}^{\infty} T_{j}\left(a_{1}-a_{2}\right) U_{j}\left(e_{1}-e_{2}\right) z^{j}
\end{aligned}
$$


Further, the identity [6,7]: $\sum_{j=0}^{\infty} T_{j}\left(a_{1}-a_{2}\right) U_{j}\left(e_{1}-e_{2}\right) z^{j}=\frac{1-2\left(a_{1}-a_{2}\right)\left(e_{1}-e_{2}\right) z+\left(2\left(a_{1}-a_{2}\right)^{2}-1\right) z^{2}}{P_{T U}}$, then

$$
\begin{aligned}
\sum_{j=0}^{\infty} T_{j+2}\left(a_{1}-a_{2}\right) U_{j}\left(e_{1}-e_{2}\right) z^{j}= & \frac{2\left(a_{1}-a_{2}\right)\left[\left(a_{1}-a_{2}\right)\left(1+z^{2}\right)-2\left(e_{1}-e_{2}\right) z\right]}{P_{T U}}- \\
& \frac{1-2\left(a_{1}-a_{2}\right)\left(e_{1}-e_{2}\right) z+\left(2\left(a_{1}-a_{2}\right)^{2}-1\right) z^{2}}{P_{T U}} .
\end{aligned}
$$

We get a new generating function defined as follows:

$$
\sum_{j=0}^{\infty} T_{j+1}\left(a_{1}-a_{2}\right) U_{j}\left(e_{1}-e_{2}\right) z^{j}=\frac{\left(a_{1}-a_{2}\right)-2\left(e_{1}-e_{2}\right) z+\left(a_{1}-a_{2}\right) z^{2}}{P_{T U}} .
$$

\section{Conclusion}

This research proposes new developments to determine generating functions. The suggested techniques are based on symmetric functions. The main results are consistent with some ideas, obtained in other previous works $[5,7,8,13]$. The first results of this work seem promising, but further investigations in the field should be continued. Future research can be done around the extension of alphabet $E$ elements and the treats of $k$ parameter values.

\section{Acknowledgements}

The authors would like to thank the anonymous referees for their valuable comments and suggestions.

\section{References}

[1] Abderrezzak, A. (1994) Généralisation de la transformation d'Euler d'une série formelle, Adv. Math., 103, 180-195.

[2] Abderrezzak, A. (1995) Généralisation d'identités de Carlitz, Howard et Lehmer, Aequationes Math., 49, 36-46.

[3] Baeder, M. A., Cohl, H. S. \& Volkmer, H. (2015) Generalizations of generating functions for higher continuous hypergeometric orthogonal polynomials in the Askey scheme, J. Math. Anal. Appl., 427, 377-398.

[4] Boussayoud, A., Abderrezzak, A. \& Kerada, M. (2015) Some applications of symmetric functions, Integers., 15, Article No. 48, 1-7.

[5] Boussayoud, A. \& Sahali, R. (2015) The application of the operator $L_{b_{1} b_{2}}^{-k}$ in the series $\sum_{j=0}^{+\infty} a_{j} b_{1}^{j} z^{j}$, J. Adv. Res. Appl. Math., 7, 68-75. 
[6] Boussayoud, A. \& Kerada, M. (2014) Symmetric and Generating Functions, Int. Electron. J. Pure Appl. Math., 7, 195-203.

[7] Boussayoud, A., Kerada, M., Sahali, R. \& Rouibah, W. (2014) Some Applications on Generating Functions, J. Concr. Appl. Math., 12, 321-330.

[8] Boussayoud, A., Abderrezzak, A. \& Kerada, M. (2013) A Generalization of Some Orthogonal Polynomials, Springer Proc. Math. Stat., 41, 229-235.

[9] Bozejko, M., Lytvynov, E. W. \&. Rodionova, I. V. (2015) An extended anyon Fock space and noncommutative Meixner-type orthogonal polynomials in infinite dimensions, Russ. Math. Surv., 70 (5), 857-899.

[10] Boubalouta, K., Boussayoud, A. \& Kerada, M. (2018) Symmetric Functions for $k$-Fibonacci Numbers and Orthogonal Polynomials, Turkish Journal of Analysis and Number Theory, 6 (3), 98-102.

[11] Cullinan, J. \& Hajir, F. (2014) On the Galois groups of Legendre polynomials, Indag. Math., $25(3), 534-552$.

[12] Doha, E. H., Abd-Elhameed, W. M. \& Bhrawy, A. H. (2013) New spectral-Galerkin algorithms for direct solution of high even-order differential equations using symmetric generalized Jacobi polynomials, Collect. Math., 64 (3), 373-394.

[13] Foata, D. \& Han, G. N. (1990) Nombres de Fibonacci et Polynômes Orthogonaux. in M. Morelli and M. Tangheroni, eds., Leonardo Fibonacci: Il Tempo, Le Opere, L'Eredita Scientica, Pacini, Rome, 179-200.

[14] Foata, D. (1977) Combinatoire et représentation du Groupe symétrique, Lect. Notes Math, Vol. 579, 339 pages.

[15] Falcon, S. (2011) On the $k$-Lucas numbers,Int. J. Contemp. Math. Sci., 21, 1039-1050.

[16] Guillemin, V. \& Sabatini, S. \& Zara, S. C. (2014) Polynomial assignments, Indag. Math., $25(5), 992-1018$.

[17] Johnston, S. J., Jooste, A. \& Jordaan, K. (2016) Quasi-orthogonality of some hypergeometric polynomials, Integral Transforms Spec. Funct., 27 (2), 111-125.

[18] Lascoux, A. (2004) Addition of \pm 1 : Application to Arithmetic. Sémin Lothar Comb., 52, $1-9$.

[19] Lascoux, A. \& Fu, A.M. (2005) Partition Analysis and Symmetrizing Operators, J. Comb. Theory, Ser. A., 109, 339-343.

[20] Macdonald, I.G. (1997) Symmetric Functions and Hall Polynomias, Oxford University Press. 
[21] Merca, M. (2014) A Generalization of the symmetry between complete and elementary symmetric functions, Indian J. Pure Appl. Math., 45 (1), 75-89.

[22] Panzone, P. A. (2012) On the generating functions of Mersenne and Fermat primes, Collect. Math., 63 (1), 59-69.

[23] Srivastava, H. M. \& Djordjević G. B. (2011) Some generating functions and other properties associated with the generalized Hermite and related polynomials, Integral Transforms Spec. Funct., 22 (12), 895-906.

[24] Stanic, M. P. (2014) Multiple orthogonal polynomials on the semicircle and applications, Appl.Math.Comput., 243, 269-282.

[25] Toufik, M. (2004) A formula for the generating functions of powers of Horadam's sequence, Australas. J. Comb., 30, 207-212. 